\title{
Special Issue Dedicated to Walter Craig
}

\section{Philippe Guyenne ${ }^{1}$ - David P. Nicholls ${ }^{2}$ - Catherine Sulem ${ }^{3}$}

Published online: 1 March 2021

(C) The Author(s), under exclusive licence to Springer Nature Switzerland AG part of Springer Nature 2021

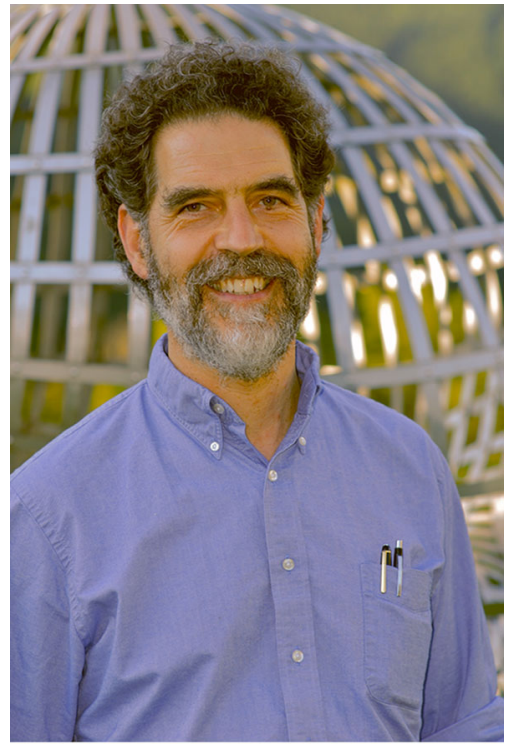

Walter Craig, Oberwolfach 2007

Photo: Jürgen Pöschel

This volume is dedicated to the memory of our dear friend and colleague Walter Craig who sadly passed away on January 18, 2019 in Hamilton, Ontario, Canada. Walter was a world renowned scholar for his work on nonlinear partial differential equations. His research included infinite-dimensional Hamiltonian systems, Schrödinger operators and spectral theory, water waves and its applications, general relativity, and cosmology.

$\bowtie \quad$ Catherine Sulem

sulem@math.utoronto.ca

1 University of Delaware, Newark, USA

2 University of Illinois at Chicago, Chicago, USA

3 University of Toronto, Toronto, Canada 
Walter authored more than 100 research articles on topics ranging from rigorous mathematical analysis to physical modeling, numerical simulations, and laboratory experiments.

Walter was born in State College, Pennsylvania on November 28, 1953 and grew up in the San Francisco Bay Area. His father William Craig was a renowned logician in the department of Philosophy at UC Berkeley. Walter received his Ph.D. under the supervision of Louis Nirenberg from the Courant Institute (NYU) in 1981. He was a postdoc at CalTech and then joined Stanford University before moving to Brown University in 1988. He was recruited to McMaster University in 2000 as a Tier 1 Canada Research Chair in Mathematical Analysis and its Applications. His contributions at McMaster University were recognized with conferral of the title Distinguished University Professor in 2016.

Walter loved water waves and was fascinated by the beauty of ocean waves. His lectures on water waves often started with poetic lines of James Joyce and ended with a picture of the Great Wave of Hokusai. His first contribution on water waves was a single-authored 200-page paper published in Communications in Partial Differential Equations in 1985 on the well-posedness of the water-wave equations and a rigorous justification of the Korteweg-de Vries (KdV) approximation. It was a groundbreaking achievement, especially back in the 1980s when there were very few rigorous results, and has remained a major reference in the mathematical theory of water waves. Another influential paper by Walter (co-authored with C. Sulem and published in the Journal of Computational Physics in 1993) proposed a numerical method for efficiently and accurately solving the full water-wave equations. It is based on a Taylor series expansion of the Dirichlet-Neumann operator and makes use of the fast Fourier transform. This approach has played a key role in the development of high-order spectral methods for simulating nonlinear water waves, which are now widely used by oceanographers and engineers.

In January 2019, Walter's new textbook "A Course on Partial Differential Equations" appeared in the AMS series "Graduate Studies in Mathematics". Aimed at beginning graduate students, it covers basic notions of partial differential equations and presents Walter's personal point of view on these topics, elegantly combining theory, phenomenology, and applications.

Walter was a mathematical analyst with a wide range of interests and an extensive scientific culture. It was common to witness him at the center of lively conversations in English or French during seminars, conferences, or workshops. In addition to being a brilliant researcher, Walter was a remarkable teacher and mentor who educated and supervised many students and postdocs. Many of them have gone on to become accomplished scholars in various countries around the world. He was generous with his time, support, and ideas, and was a constant source of inspiration, whose enthusiasm and friendship never waned.

Walter was a devoted and loving husband to Deirdre Haskell and a proud father of Zoe Haskell-Craig. He will be greatly missed by all who had the privilege of knowing him as a mathematician, colleague, collaborator, and dear friend. A memorial tribute entitled "Remembrances of Walter Craig" has appeared in the Notices of the American Mathematical Society, Volume 67, no. 4 (2020), 520-531. 
The present volume is composed of 11 articles by various researchers working on water waves who were Walter's mentees, collaborators, or were strongly influenced by his work. It covers topics at the heart of Walter's interests including mathematical analysis of the water-wave equations and related systems, asymptotic models in different scaling regimes, and traveling wave solutions.

The article by Berti, Feola, and Pusateri [5] is a review of a rigorous reduction to cubic Birkhoff normal form for the two-dimensional gravity water-wave problem with a periodic free surface on infinite depth. This result confirms a conjecture by Dyachenko and Zakharov (1994) and Craig and Wolfork (1995) based on the formal Birkhoff integrability of the water-wave Hamiltonian truncated at fourth order. They also prove that small periodic perturbations of the flat surface lead to a solution that remains regular and small for suitably long times. Berti, Feola, and Franzoi [4] extend this result to the two-dimensional gravity-capillary water-wave problem with a periodic free surface on arbitrary depth. The normal form is nontrivial in this case due to the possible presence of three-wave resonances. Lushnikov and Zakharov [10] provide necessary conditions for the existence and persistence of complex singularities for the initial value problem for water waves. This question has proven to be important in several fluid interface problems. Alazard [2] introduces a simple compact elliptic formulation of the Hele-Shaw equations in the spirit of the Zakharov-Craig-Sulem formulation for the water-wave problem. Based on this formulation, he identifies several properties of the Hele-Shaw problem, which are related to convexity, including the existence of a large class of strong Lyapunov functions. Desjardins, Lannes, and Saut [7] address the question of well-posedness of equations which model a continuously stratified ocean. They investigate perturbations of a stable vertical stratification and show that solutions exist locally in time.

The following papers are devoted to various asymptotic models for the waterwave problem. Bambusi [3] uses a Hamiltonian normal form approach to study the water-wave problem in the small-amplitude long-wave regime. He shows that the normal form up to fifth order in the dispersion parameter consists of two decoupled equations, one describing right-moving waves and the other describing left-moving waves. Each of these equations is integrable, being a linear combination of the first three equations in the KdV hierarchy. Craig, Guyenne, and Sulem [6] propose a Hamiltonian version of Dysthe's equation for two-dimensional weakly modulated gravity waves on deep water. Their derivation relies on a Birkhoff normal form transformation that eliminates all non-resonant cubic terms and leads to a non-perturbative procedure for the surface reconstruction. Very good agreement is found in comparison to direct numerical simulations on the Benjamin-Feir instability of Stokes waves. Duchêne and Iguchi [8] show that the Isobe-Kakinuma model for water waves admits a Hamiltonian structure and its Hamiltonian is a higher order shallow water approximation to the one of the full water-wave problem. Rybkin, Nicolsky, Pelinovsky, and Buckel [11] study nonlinear shallow water waves on an inclined channel using the generalized Carrier-Greenspan hodograph transform. In particular, they show how some restrictive assumptions on the size of the initial velocity and boundary conditions can be removed in their method of data projection.

The two last papers concern traveling wave solutions. Groves [9] presents a fixedpoint approach to the existence of solitary water waves with both weak and strong 
surface tension. This is an alternative approach in which one works directly with the Zakharov-Craig-Sulem formulation of the water-wave problem. Akers and Nicholls [1] study the question of existence of branches of traveling waves in weakly nonlinear models in the presence of non-simple bifurcation. They show the existence and analytic dependence of branches of not only Stokes waves (simple bifurcation) but also Wilton ripples (bifurcation from a speed with a two-dimensional null space).

\section{References}

1. Akers, B., Nicholls, D.P.: Wilton ripples in weakly nonlinear dispersive models of water waves: existence and analyticity of solution branches. Water Waves 3 (2021)

2. Alazard, T.: Convexity and the Hele-Shaw Equation. Water Waves 3 (2021)

3. Bambusi, D.: Hamiltonian studies on counter-propagating water waves. Water Waves 3 (2021)

4. Berti, M., Feola, R., Franzoi, L.: Quadratic life span of periodic gravity-capillary water waves. Water Waves 3 (2021)

5. Berti, M., Feola, R., Pusateri, F.: Birkhoff normal form for gravity water waves. Water Waves 3 (2021)

6. Craig, W., Guyenne, P., Sulem, C.: Normal form transformations and Dysthe's equation for the nonlinear modulation of deep-water gravity waves. Water Waves 3 (2021)

7. Desjardins, B., Lannes, D., Saut, J.-C.: Normal mode decomposition and dispersive and nonlinear mixing in stratified fluids. Water Waves 3 (2021)

8. Duchene, V., Iguchi, T.: A Hamiltonian structure of the Isobe-Kakinuma model for water waves. Water Waves 3 (2021)

9. Groves, M.D.: An existence theory for gravity-capillary solitary waves. Water Waves, 3 (2021)

10. Lushnikov, P.M., Zakharov, V.E.: Poles and branch cuts in free surface hydrodynamics. Water Waves 3 (2021)

11. Rybkin, A., Nicolsky, D., Pelinovsky, E., Buckel, M.: The generalized Carrier-Greenspan transform for the shallow water system with arbitrary initial and boundary conditions. Water Waves 3 (2021)

Publisher's Note Springer Nature remains neutral with regard to jurisdictional claims in published maps and institutional affiliations. 